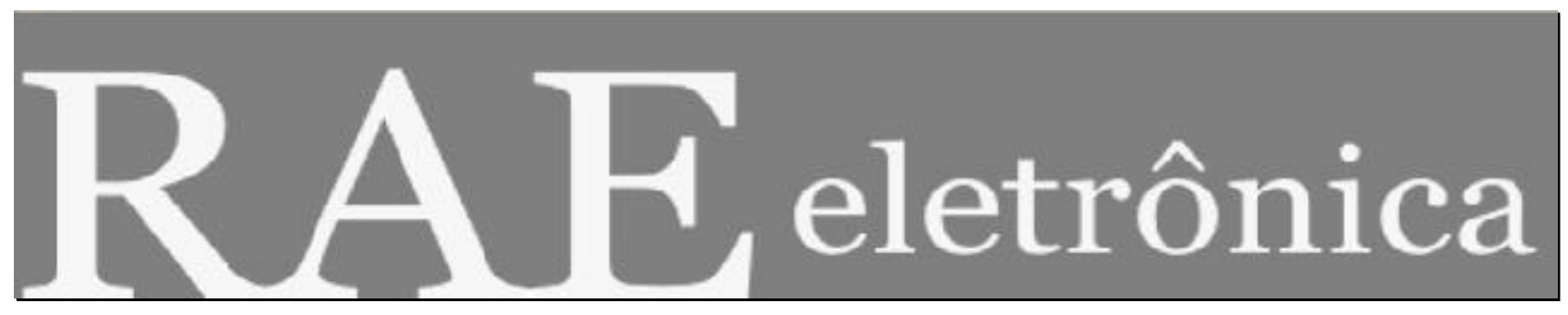

FÓRUM - A TEORIA INSTITUCIONAL EM UM CONTEXTO BRASILEIRO:

DINÂMICAS DE INOVAÇÃO E IMITAÇÃO

\title{
TEORIA INSTITUCIONAL E DEPENDÊNCIA DE RECURSOS NA ADAPTAÇÃO ORGANIZACIONAL: UMA VISÃO COMPLEMENTAR
}

Por:

\section{Carlos Ricardo Rossetto \\ Adriana Marques Rossetto}

RAE-eletrônica, v. 4, n. 1, Art. 7, jan./jul. 2005

http://www.rae.com.br/eletronica/index.cfm?FuseAction=Artigo\&ID=1869\&Secao=FOR.TE.INS\&Vol ume $=4 \&$ Numero $=1 \&$ Ano $=2005$

CCopyright, 2005, RAE-eletrônica. Todos os direitos, inclusive de tradução, são reservados. É permitido citar parte de artigos sem autorização prévia desde que seja identificada a fonte. A reprodução total de artigos é proibida. Os artigos só devem ser usados para uso pessoal e nãocomercial. Em caso de dúvidas, consulte a redação: raeredacao@fgvsp.br.

A RAE-eletrônica é a revista on-line da FGV-EAESP, totalmente aberta e criada com o objetivo de agilizar a veiculação de trabalhos inéditos. Lançada em janeiro de 2002, com perfil acadêmico, é dedicada a professores, pesquisadores e estudantes. Para mais informações consulte o site www.rae.com.br/eletronica.

\section{RAE-eletrônica}

ISSN 1676-5648

(C2005 Fundação Getulio Vargas - Escola de Administração

de Empresas de São Paulo.

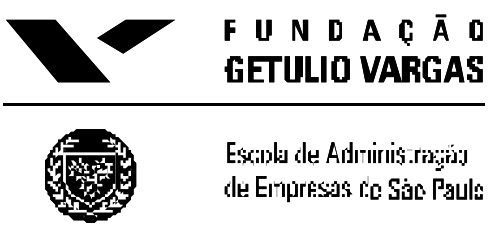




\title{
FÓRUM - A TEORIA INSTITUCIONAL EM UM CONTEXTO BRASILEIRO: DINÂMICAS DE INOVAÇÃO E IMITAÇÃO - TEORIA INSTITUCIONAL E DEPENDÊNCIA DE RECURSOS NA ADAPTAÇÃO ORGANIZACIONAL: UMA VISÃO COMPLEMENTAR \\ Carlos Ricardo Rossetto - Adriana Marques Rossetto
}

\section{RESUMO}

O presente trabalho aborda o processo de adaptação estratégica organizacional utilizando para sua análise duas abordagens distintas: a perspectiva Institucional e da Dependência de Recursos. O texto em um primeiro momento apresenta uma revisão bibliográfica sobre as perspectivas e após a discussão de como a utilização de ambas na análise dos processos pode contribuir para seu entendimento. Apesar das diferenças entre as perspectivas, os autores, na revisão bibliográfica feita, concluem que elas podem ser utilizadas de forma complementar. A ponte de complementaridade decorre de suas concepções a respeito do meio ambiente, que o considera o fator chave do funcionamento organizacional. A perspectiva Institucional afirma que as organizações resistem às pressões do ambiente na extensão em que a tradição das empresas, o contexto ambiental, ou ambos, suportam tal resistência. Já a perspectiva da Dependência de Recursos sugere que as organizações adaptam-se às pressões na extensão em que os atores organizacionais corretamente percebem e gerenciam as mudanças necessárias. Portanto, para a compreensão de como os processos organizacionais que resultam na sobrevivência ou não das organizações ocorrem há que se considerar a visão das duas perspectivas.

\section{PALAVRAS-CHAVE}

Perspectiva institucional adaptação estratégica, dependência de recursos, determinismo ambiental, isomorfismo.

\begin{abstract}
This paper discusses the analysis of the process of strategic adaptation from two perspectives: Institutional Analysis and Resource Dependence. A review of the literature is presented followed by a discussion about the contribution of both perspectives for the understanding of the strategic adaptation process of organizations. The authors argue that, despite their apparent differences, they can be complimentary. This is mostly due to their common view of the environment as the key factor to organizational functioning. According to the Institutional Perspective, organizations are resistant to organizational pressures to the extent that organizational tradition, environment context, or both, support this resistance. On the other hand, Resource Dependency suggests organizations adapt to such pressure to the extent that organizational actors correctly perceive and manage the necessary changes. Hence, to comprehend how organizational processes result in survival or not, both perspectives need to be considered in the analysis of strategic decisions.
\end{abstract}

\section{KEY WORDS}

Institutional analysis, strategic adaptation, resources dependence, environmental determinism, isomorfism. 


\section{FÓRUM - A TEORIA INSTITUCIONAL EM UM CONTEXTO BRASILEIRO: DINÂMICAS DE INOVAÇÃO E IMITAÇÃO - TEORIA INSTITUCIONAL E DEPENDÊNCIA DE RECURSOS NA ADAPTAÇÃO ORGANIZACIONAL: UMA VISÃO COMPLEMENTAR \\ Carlos Ricardo Rossetto - Adriana Marques Rossetto}

\section{INTRODUÇÃO}

Por muitos anos, adaptação às mudanças do ambiente tem sido uma área central do estudo das organizações (GINSBERG; BUCHHOLTZ, 1990; HREBINIAK; JOYCE, 1985). Como as organizações vivem cada vez mais em ambientes dinâmicos, os estudos do processo de adaptação estratégica organizacional têm sido enfatizados como de fundamental importância pelos teóricos das organizações.

Enquanto a literatura conceitual sobre adaptação organizacional tem geralmente assumido que mudanças ambientais levam à mudança organizacional, esta questão não tem recebido grande atenção da investigação empírica (BOEKER; GOODSTEIN, 1991; ZAJAC; SHORTELL, 1989).

O processo de adaptação às contínuas mudanças no ambiente da organização, chamado de administração estratégica (SCHENDEL e HOFER, 1979), exige dos gerentes não somente enfrentar as mudanças ocorridas no ambiente da organização, mas também lidar com mudanças causadas pelos processos internos da organização (GREINER, 1972).

Embora teóricos da estratégia organizacional como Andrews (1971), Chaffee (1985), Child (1972), Miles e Cameron (1982), Schendel e Hofer (1979), Jennings e Seaman (1994) sugiram que os gerentes organizacionais mudam suas estratégias para refletir as mudanças ras condições de seus ambientes, outros como Boeker (1989), Hannan e Freeman (1984), Kelly e Amburgey (1991), Pfeffer e Salancik (1978), Quinn (1980), apud Jennings e Seaman (1994) têm afirmado que as organizações são restringidas na sua habilidade para adaptarem-se.

Portanto, o debate existente entre os estudiosos do processo de adaptação estratégica tem se centrado, fundamentalmente, em dois aspectos: a) a visão determinista que considera o ambiente como elemento principal na definição das estratégias orga nizacionais e, b) a visão voluntarista ou da escolha estratégica que defende a organização como tendo capacidade de manipular o ambiente em função de suas capacidades materiais e organizacionais. (ROSSETTO, 1998; ROSSETTO; ROSSETTO, 2001; ROSSETTO; ROSSETTO, 2002; ROSSETTO; SALVADOR; MELLO, 2002; ROSSETTO; CASTRO, 2003).

Estes dois aspectos são de fundamental importância para o entendimento dos pressupostos das perspectivas abordadas neste texto e serão mais bem explicados a seguir.

\section{DETERMINISMO VERSUS VOLUNTARISMO}

Como se descreveu, o processo de adaptação estratégica organizacional tem sido discutido e explicado por diversos teóricos organizacionais. O questionamento central na recente discussão da adaptação estratégica organizacional é se ela deriva do aspecto gerencial ou ambiental (ASTLEY; VAN DE VEN, 1983). 


\section{FÓRUM - A TEORIA INSTITUCIONAL EM UM CONTEXTO BRASILEIRO: DINÂMICAS DE INOVAÇÃO E IMITAÇÃO - TEORIA INSTITUCIONAL E DEPENDÊNCIA DE RECURSOS NA ADAPTAÇÃO ORGANIZACIONAL: UMA VISÃO COMPLEMENTAR \\ Carlos Ricardo Rossetto - Adriana Marques Rossetto}

A questão é uma visão de adaptação como um processo refletindo escolha ou seleção versus outra como uma reação necessária às forças ambientais (CHILD, 1972; ALDRICH, 1979), ou seja, a predominância do voluntarismo ou do determinismo externo no processo de mudança estratégica (HANNAN; FREEMAN, 1977).

O determinismo ambiental e o voluntarismo ou escolha estratégica são vistos como mutuamente exclusivos, isto é, ou só o ambiente determina as ações da organização ou só a percepção dos gerentes é que define as escolhas estratégicas. Como afirmam Hrerbiniak e Joyce (1985) as principais abordagens para a questão da adaptação estratégica enfatizam os fins mutuamente exclusivos e diferenciados do determinismo ambiental e do voluntarismo (HREBINIAK; JOYCE, 1985).

Astley e Van de Ven (1983) concluíram que o interesse das questões de pesquisa a respeito de organizações complexas deveria em primeiro lugar admitir ambas as visões: determinista e voluntarista; e segundo justapor estas visões para estudar as interações e interdependências recíprocas existentes nos processos de adaptação organizacional.

Na visão de Miles (1980) a questão do determinismo e do voluntarismo tem que ser reformulada do "se?" para "quanto?" pois em quase todas as instâncias organizacionais os elementos da vontade e do determinismo estarão presentes.

A preocupação básica das organizações, partindo da visão determinista do ambiente, está no ajuste entre suas estruturas e processos e o ambiente (DILL, BURNS e STALKER, LAWRENCE e LORSCH e MORSE apud MILES, 1980). Grande parte das pesquisas feitas utilizando a visão contingencialista, ignora os processos pelos quais a adaptação organizacional ocorre (MILES, 1980). Para ela a função dos gerentes está apenas em adequar as estruturas organizacionais às condições mutáveis do ambiente, desconsiderando os processos pelos quais eles fazem suas escolhas e as implantam.

Antagônica a esta visão do determinismo ambiental está a visão voluntarista formada por um conjunto de perspectivas que tratam os padrões de mudança das organizações como conseqüência das respostas dos executivos às mudanças ambientais. Ao adotarem o conceito de escolha estratégica, estas perspectivas compartilham da idéia de que existe um espaço de decisão no qual é possível se decidir a estratégia que definirá os rumos da organização e que o ambiente, apesar de funcionar como uma restrição, não elimina este espaço (CUNHA, 1996; ROSSETTO; ROSSETTO; MELLO, 2002; ROSSETTO; ROSSETTO, 2003).

Nesta visão, a adaptação organizacional refere-se à habilidade dos administradores em reconhecer, interpretar e implementar estratégias, de acordo com as necessidades e mudanças percebidas no seu ambiente, de forma a assegurar suas vantagens competitivas. Como se pode notar, o estudo do processo de adaptação estratégica envolve as visões deterministas do ambiente organizacional e a voluntarista da escolha das estratégias pelos tomadores de decisão nas organizações.

As teorias formadas a partir destas visões serviram de pressupostos para que surgissem abordagens distintas sobre os processos de adaptação estratégica organizacional. Desta forma, duas linhas teóricas serão alvo da presente discussão: a da perspectiva Institucional que utiliza como pressuposto a visão determinista para explicar as ações organizacionais frente o ambiente e a da perspectiva da Dependência de Recursos cuja visão voluntarista é a que prevalece. Com o intuito de facilitar a compreensão sobre o objeto principal do estudo, que consiste em discutir o papel destas perspectivas no entendimento dos processos organizacional, far-se-á a seguir uma descrição das mesmas. 


\section{FÓRUM - A TEORIA INSTITUCIONAL EM UM CONTEXTO BRASILEIRO: DINÂMICAS DE INOVAÇÃO E IMITAÇÃO - TEORIA INSTITUCIONAL E DEPENDÊNCIA DE RECURSOS NA ADAPTAÇÃO ORGANIZACIONAL: UMA VISÃO COMPLEMENTAR \\ Carlos Ricardo Rossetto - Adriana Marques Rossetto}

\section{A PERSPECTIVA INSTITUCIONAL}

Versões iniciais da teoria institucional colocaram ênfase particular sobre o caráter legitimado das regras institucionais, mitos e crenças moldando a realidade social e sobre o processo pelo qual organizações tendem a tornar-se impregnadas de valor e significado social (BERGER; LUCKMANN, 1967; SELZNICK, 1949, 1957). Estudos da institucionalização têm apresentado um conjunto de influências que os mesmos exercem sobre as características das organizações (MEYER; SCOTT; DEAL, 1983; MEYER; SCOTT; STRANG, 1987; SCOTT, 1987a; SINGH, TUCKER; HOUSE, 1986) e sobre a mudança organizacional (HININGS; GREENWOOD, 1988; TOLBERT; ZUCKER, 1983).

A perspectiva institucional para o estudo das organizações pode ser tipificada como uma abordagem simbólico-interpretativa da realidade organizacional, apresentando uma posição epistemológica predominantemente subjetivista, na qual é salientada a construção social da realidade organizacional (PRATES, 2000, p. 90).

Esta perspectiva busca explicar os fenômenos organizacionais, através do entendimento do como e do por que as estruturas e processos organizacionais tornam-se legitimados e suas conseqüências nos resultados alcançados (MEYER; ROWAN, 1977; SELZNICK, 1949, 1957, 1996; ZUCKER, 1987; DANIELS; JOHNSON; CHERNATONY, 2002).

A visão institucional vê as organizações como tendo duas dimensões essenciais: a técnica e a institucional.

$\mathrm{O}$ ambiente técnico caracteriza-se pela troca de bens e serviços, enquanto o ambiente institucional conduz o estabelecimento e a difusão de normas de atuação, necessárias ao alcance da legitimidade organizacional. Deste modo, organizações submetidas a pressões do ambiente técnico e do ambiente institucional são avaliadas, respectivamente, pela eficiência e pela adequação às exigências sociais. (MACHADO-DA-SILVA; FONSECA; FERNANDES, 1999, p. 113).

Discussões dos ambientes institucionais (HIRSCH, 1972; MCNEIL; MINIHAN, 1977; ZALD, 1978) e seus efeitos sobre as estruturas e processos organizacionais (DOWLING; PFEFFER, 1975; HIRSCH, 1975; MEYER; ROWAN, 1977) têm demonstrado que a escola institucional é um suporte vital na pesquisa corrente sobre a adaptação organizacional.

A proposta desta abordagem é que deve ser acrescentado um sistema de crenças e de normas institucionalizadas à visão de ambiente, que é formado em sua totalidade por fluxos e intercâmbios técnicos. Desta forma, o ambiente institucional deve ser entendido como um rico contexto que compreende o ambiente técnico ampliado ao domínio do simbólico (CARVALHO; VIEIRA; LOPES, 1999).

Portanto, já que as organizações adotam determinadas estruturas e procedimentos transferidos pelos costumes e normas aceitas coletivamente a fim de alcançarem a legitimidade, percebe-se que surge um 


\section{FÓRUM - A TEORIA INSTITUCIONAL EM UM CONTEXTO BRASILEIRO: DINÂMICAS DE INOVAÇÃO E IMITAÇÃO - TEORIA INSTITUCIONAL E DEPENDÊNCIA DE RECURSOS NA ADAPTAÇÃO ORGANIZACIONAL: UMA VISÃO COMPLEMENTAR \\ Carlos Ricardo Rossetto - Adriana Marques Rossetto}

conflito, no que tange à tomada decisão, entre a desejada legitimidade e as exigências técnicas que têm como fator principal a eficiência. O que acontece geralmente é que a organização age a fim de garantir a sua legitimidade institucional, indo de encontro às rígidas medidas de eficiência técnica (CARVALHO, VIEIRA; LOPES, 1999; MELO; OLIVEIRA, 2003; ALVES; KOGA, 2003; ANDRADE; MESQUITA, 2003).

Machado-da-Silva e Gonçalves (1999) ressaltam que as organizações interagem com seu ambiente a procura de legitimação. Dessa forma, as estratégias escolhidas e as decisões empreendidas são influenciadas por uma inércia ambiental, no que concerne a conformidade às regras, às normas e às crenças (mitos) institucionalizadas, em conflito com critérios de eficiência. Este posicionamento gera gaps entre a estrutura formal e as práticas realmente adotadas.

Embora existam diversas escolas do pensamento dentro desta perspectiva (SCOTT, 1987b), muito da literatura institucional utiliza o conceito de isomorfismo para explicar a forma como as características organizacionais são modificadas para aumentar a compatibilidade com as características ambientais (DIMAGGIO; POWELL, 1983; ROWAN, 1982). O isomorfismo pressupõe que as organizações respondem de maneira similar a outras organizações que estão de alguma forma ajustadas ao ambiente.

O que leva as organizações a assumirem esta postura isomórfica em relação às organizações líderes no seu ambiente específico é o fato delas buscarem uma autodefesa em relação aos problemas que não conseguem resolver com idéias criadas por elas próprias. Assim passam a desenvolver processos semelhantes aos observados em outras organizações a fim de facilitar as suas relações interorganizacionais, favorecendo o seu funcionamento a partir da utilização de regras socialmente aceitas (MACHADO-DA-SILVA; FONSECA, 1993).

DiMaggio e Powell (1983) argumentam que o isomorfismo institucional é a razão dominante pela qual as organizações assumem determinadas formas. De acordo com os autores, as forças motoras que levam à racionalização e à burocratização se baseiam em uma economia capitalista de mercado, tendo a burocratização como uma jaula de ferro em que a humanidade se vê confinada.

O isomorfismo é um conjunto de restrições que forçam uma unidade de uma população a parecer-se com outras unidades que se colocam em um mesmo conjunto de condições ambientais. Tal abordagem sugere que as características organizacionais são modificadas na direção do aumento de compatibilidade com as características ambientais; o número de organizações em uma população é função da capacidade ambiental projetada e a diversidade das formas organizacionais é isomórfica à diversidade ambiental.

Seguindo Meyer (1979) e Fennell (1980), DiMaggio e Powell (1983) argumentam que existem dois tipos de isomorfismo: o competitivo e o institucional. Hannan e Freeman (1977), tratam do isomorfismo competitivo assumindo um sistema de racionalidade que enfatiza o mercado de competição e a mudança de nicho, entre outras. Esta visão sugere que isto é mais apropriado para aqueles campos onde existe competição livre e aberta.

DiMaggio e Powell (1983) identificam três mecanismos através do qual a mudança isomórfica institucional ocorre, cada um com seus próprios antecedentes: 1) isomorfismo coercivo; 2) isomorfismo mimético; e, 3) isomorfismo normativo. 


\section{FÓRUM - A TEORIA INSTITUCIONAL EM UM CONTEXTO BRASILEIRO: DINÂMICAS DE INOVAÇÃO E IMITAÇÃO - TEORIA INSTITUCIONAL E DEPENDÊNCIA DE RECURSOS NA ADAPTAÇÃO ORGANIZACIONAL: UMA VISÃO COMPLEMENTAR \\ Carlos Ricardo Rossetto - Adriana Marques Rossetto}

Em primeiro lugar estão as forças coercitivas do ambiente, tais como, as regulamentações governamentais e as expectativas culturais capazes de impor uniformidades às organizações. As regulamentações governamentais que, por exemplo, obrigam os restaurantes a manter uma ala, em suas dependências para os não fumantes.

Como Meyer e Rowan (1977) sugeriram, as organizações adotam formas que são institucionalizadas e legitimadas pelo Estado. Em algumas circunstâncias, a mudança organizacional é uma resposta direta ao mandato do governo: adotar novos controles de poluição, manter contabilidade não lucrativa, modificam processos influenciados por legislações ambientais.

Nem todo o isomorfismo institucional deriva, entretanto, da autoridade coercitiva. A incerteza é uma poderosa força que encoraja imitação. Quando os objetivos são ambíguos ou quando o ambiente cria incerteza simbólica, as organizações modelam-se em outras organizações. Em geral, quanto maior o número de empregados ou consumidores servidos por uma organização, mais fortes as pressões sofridas por ela para fornecer programas e serviços oferecidos por outras organizações. Então, ou uma força de trabalho bem treinada ou uma ampla base de consumidor deve encorajar o isomorfismo mimético. Estudo feito por Redmond (2003) sobre inovação, difusão e mudança institucional mostra que as organizações não escolhem formas diferentes daquelas já instituídas. Ele afirma que a proporção de empresas que adotam formas diferentes de inovação é muito pequena e que a maioria age conforme o ambiente institucional.

A terceira fonte de mudança organizacional isomórfica é normativa e origina-se primariamente a profissionalização. Seguindo Larson (1977) e Collins (1979), DiMaggio e Powell (1983) interpretam profissionalização como a luta coletiva dos membros de uma ocupação para definir condições e métodos de seus trabalhos e para estabelecer uma base cognitiva e legitimação para sua autonomia ocupacional.

As profissões estão sujeitas às mesmas pressões cognitivas e miméticas que as organizações. Além disso, enquanto vários tipos de profissionais dentro de uma organização devem diferenciar-se uns dos outros, eles exibem muita similaridade com suas contrapartes profissionais em outras organizações. Em adição, em muitos casos, o poder profissional é tanto fixado pelo Estado como é criado pelas atividades das profissões.

A perspectiva institucional, em conseqüência, concebe o desenho organizacional não como um processo racional, e sim como processo derivado das pressões tanto externas como internas que, com o tempo, levam às organizações a se parecerem uma com as outras. De acordo com esta perspectiva, as escolhas estratégicas ou as intenções de controle seriam originadas na ordem institucional na qual uma organização se vê imersa.

Em suma, a perspectiva institucional é uma estrutura determinística que coloca grande ênfase sobre as normas do ambiente e o peso da história da empresa para explicar as ações organizacionais (EISENHARDT, 1988). 


\title{
FÓRUM - A TEORIA INSTITUCIONAL EM UM CONTEXTO BRASILEIRO: DINÂMICAS DE INOVAÇÃO E IMITAÇÃO - TEORIA INSTITUCIONAL E DEPENDÊNCIA DE RECURSOS NA ADAPTAÇÃO ORGANIZACIONAL: UMA VISÃO COMPLEMENTAR \\ Carlos Ricardo Rossetto - Adriana Marques Rossetto
}

A PERSPECTIVA DA DEPENDÊNCIA DE RECURSOS

\begin{abstract}
Aldrich e Pfeffer (1976) sugerem que existe uma perspectiva alternativa à institucional que eles denominaram de Dependência de Recursos, a qual considera o ambiente como fonte de influência nas organizações, mas de uma maneira diferente. A premissa básica da perspectiva da Dependência de Recursos é que as decisões são tomadas dentro das organizações, ou seja, dentro do contexto político interno das mesmas, e se relacionam com condições ambientais enfrentadas por elas. Nela o gerenciamento das relações externas é a chave para a sobrevivência organizacional, e é fortemente influenciada pelas forças externas (ALDRICH; PFEFFER, 1976; PFEFFER; SALANCIK, 1978).
\end{abstract}

A perspectiva da Dependência de Recursos reconhece os efeitos do ambiente sobre os resultados das estratégias, mas também se concentra no papel da gerência em captar recursos para obter performance satisfatória. A capacidade organizacional para obter recursos vitais e a negociação e o relacionamento interorganizacional também constituem duas atividades fundamentais nesta perspectiva que admite que certas mudanças no meio ambiente ocorrem, em parte, pela determinação dos administradores organizacionais, porque estes se preocupam em compatibilizar as características do ambiente aos interesses específicos das suas organizações.

Outro aspecto importante desta perspectiva, é que as organizações tentam se relacionar ativamente com o ambiente, manipulando-o para seu próprio benefício. Em lugar de assumir um papel passivo das forças ambientais, as organizações tomam decisões estratégicas para adaptarem-se ao ambiente.

Um elemento chave na perspectiva da Dependência de Recursos é a escolha estratégica (CHANDLER, 1962; CHILD, 1972). A perspectiva da escolha estratégica (CHILD, 1972) atribui às ações tomadas pelos membros organizacionais para adaptarem as organizações a um ambiente, os resultados organizacionais.

Estes autores argúem que propostas de ações proliferam nas organizações e que os membros organizacionais têm substancial liberdade de escolher a melhor entre elas. A perspectiva focaliza atenção sobre os indivíduos e grupos dentro da organização, para explicar os processos organizacionais. Este foco sobre o comportamento assume que os atores organizacionais possuem a discrição de agir por sua própria vontade (HAMBRICK; FINKELSTEIN, 1987; STATA, 2002; ABIDIN; TAYLOR, 2002).

A perspectiva da Escolha Estratégica enfatiza as explicações não determinísticas dos processos e resultados organizacionais (BOURGEOIS, 1984). Aldrich e Pfeffer (1976), com base na obra de Child (1972), observam que existem três maneiras nas quais as escolhas estratégicas operam em relação ao ambiente. A primeira é que quem toma as decisões tem autonomia. Esta autonomia é muito maior do que seria sugerido por uma estrita aderência ao determinismo ambiental. A autonomia de quem toma as decisões refere-se ao fato de que mais de uma decisão pode ser feita acerca do nicho ambiental que é ocupado - mais de uma estrutura é apropriada para um determinado ambiente.

A segunda maneira das escolhas estratégicas operarem com o ambiente, é quando existe a intenção de manipular o mesmo. As organizações tentam criar demanda para seus produtos ou podem tentar entrar em acordo com outras organizações a fim de regular a concorrência, legal ou ilegalmente. Operando através de processos políticos, as organizações podem também assegurar a aprovação de tarifas e quotas para limitar a concorrência por parte de organizações estrangeiras. 


\section{FÓRUM - A TEORIA INSTITUCIONAL EM UM CONTEXTO BRASILEIRO: DINÂMICAS DE INOVAÇÃO E IMITAÇÃO - TEORIA INSTITUCIONAL E DEPENDÊNCIA DE RECURSOS NA ADAPTAÇÃO ORGANIZACIONAL: UMA VISÃO COMPLEMENTAR \\ Carlos Ricardo Rossetto - Adriana Marques Rossetto}

A terceira maneira pela qual se tomam as decisões estratégicas acerca do ambiente se baseia no fato de que as condições ambientais particulares são percebidas e avaliadas de maneira diferente por diferentes pessoas. Os atores organizacionais definem a realidade em termos de sua história e valores próprios. Kanter (1983) tem estudado as políticas de recrutamento de executivos em uma grande empresa, e observou como resultado a seleção de executivos com antecedentes muito homogêneos. Kanter sugere que isto permite a confiança mútua, pois os decisores experimentam as coisas da mesma maneira e tomam os mesmos tipos de decisões.

O ambiente é percebido, interpretado e avaliado por atores humanos dentro das organizações. A percepção se transforma em realidade e as condições ambientais são importantes somente na medida em que são percebidas por aqueles que tomam as decisões. Diferentes atores podem perceber o mesmo fenômeno de maneiras completamente distintas. O ambiente é atualizado pelos tomadores de decisão com base em suas percepções, interpretações e avaliações.

Assim, distintas organizações atuam de forma diferente frente às mesmas condições ambientais, se as percepções de tais condições são distintas. A este respeito, Starbuck (1971) assinalou que a questão crítica é o quanto as percepções organizacionais diferem dos indicadores objetivos das condições ambientais.

O modelo de tomada de decisão da perspectiva da Dependência de Recursos sugere que as organizações são, ou tentam ser, ativas para influenciar seus ambientes. Este aspecto contribui para a variação entre as organizações, dado que as mesmas são o resultado das respostas conscientemente planejadas das contingências ambientais. As organizações tentam absorver a interdependência e a incerteza, como através de fusões de organizações (PFEFFER, 1972b), de maneira parcial através da cooperação (Pfeffer, 1972a) ou através do movimento de pessoal entre organizações (PFEFFER; LEBLEBICI, 1973; ALDRICH; PFEFFER, 1976; FROOMAN, 1999).

Em suma, a perspectiva da dependência de Recursos é uma estrutura teórica com relativo voluntarismo, que coloca ênfase na maneira pela qual as organizações tratam das contingências do ambiente. Teóricos da dependência de recursos dizem que a organização luta contra as ameaças externas e a falta de recursos e ressalta o componente político e interorganizacional do processo de aquisição destes recursos.

\section{ANÁLISE COMPARATIVA DAS PERSPECTIVAS INSTITUCIONAL E DA DEPENDÊNCIA DE RECURSOS}

Apesar da contribuição de ambas as abordagens para a consolidação das teorias sobre adaptação estratégica organizacional, muitos processos que ocorrem nas organizações inseridas em ambientes dinâmicos como os que hoje vivenciam, ficam de certa forma pouco entendidos se utilizarmos como base para as análises apenas uma das perspectivas. Desta forma, a proposição feita a partir de uma análise comparativa entre as duas perspectivas é que identificadas as convergências e as divergências entre elas, as mesmas possam ser utilizadas de forma compementar no estudo dos processos de adaptação das organizações. 
FÓRUM - A TEORIA INSTITUCIONAL EM UM CONTEXTO BRASILEIRO: DINÂMICAS DE INOVAÇÃO E IMITAÇÃO - TEORIA INSTITUCIONAL E DEPENDÊNCIA DE RECURSOS NA ADAPTAÇÃO ORGANIZACIONAL: UMA VISÃO COMPLEMENTAR

Carlos Ricardo Rossetto- Adriana Marques Rossetto

As suposições convergentes e divergentes entre a perspectiva Institucional e a da Dependência de Recursos, conforme Quadro 1, são relevantes para a caracterização das respostas estratégicas a pressões e expectativas externas.

Quadro 1 - Comparação entre a perspectiva institucional e da dependência de recursos

\begin{tabular}{|c|c|c|c|}
\hline \multirow{2}{*}{$\begin{array}{l}\text { FATOR DE } \\
\text { EXPLICAÇÃO }\end{array}$} & \multirow{2}{*}{$\begin{array}{l}\text { SUPOSIÇÕES } \\
\text { CONVERGENTES }\end{array}$} & \multicolumn{2}{|l|}{ FOCO DIVERGENTE } \\
\hline & & $\begin{array}{l}\text { PERSPECTIVA } \\
\text { INSTITUCIONAL }\end{array}$ & \begin{tabular}{|ll} 
PERSPECTIVA & DA \\
DEPENDÊNCIA & DE \\
RECURSOS &
\end{tabular} \\
\hline \multirow[t]{6}{*}{$\begin{array}{l}\text { CONTEXTO DO } \\
\text { COMPORTAMENTO } \\
\text { ORGANIZACIONAL }\end{array}$} & $\begin{array}{l}\text { Escolha organizacional } \\
\text { é restringida pelas } \\
\text { múltiplas pressões } \\
\text { externas } \\
\text { Os ambientes } \\
\text { organizacionais são } \\
\text { coletivos e } \\
\text { interconectados }\end{array}$ & $\begin{array}{l}\text { Ambiente institucional } \\
\text { com nenhum } \\
\text { comportamento de } \\
\text { escolha } \\
\text { Acomodando-se a } \\
\text { normas coletivas }\end{array}$ & $\begin{array}{l}\text { Ambiente da tarefa } \\
\text { com comportamento } \\
\text { de escolha ativo } \\
\text { Lidando com } \\
\text { interdependências }\end{array}$ \\
\hline & Inerconectados & Pressões invisíveis & Pressões visíveis \\
\hline & A sobrevivência & Isomorfismo & Adaptação \\
\hline & $\begin{array}{l}\text { Organizacional } \\
\text { depende da } \\
\text { sensibilidade às } \\
\text { demandas e } \\
\text { expectativas externas }\end{array}$ & $\begin{array}{l}\text { Aderência à regras e } \\
\text { normas }\end{array}$ & $\begin{array}{l}\text { Administração da } \\
\text { escassez de recursos }\end{array}$ \\
\hline & $\begin{array}{l}\text { As organizações } \\
\text { buscam estabilidade e } \\
\text { previsibilidade }\end{array}$ & $\begin{array}{l}\text { Persistência } \\
\text { organizacional }\end{array}$ & Redução da incerteza \\
\hline & & $\begin{array}{l}\text { Hábito e convenções } \\
\text { de mais valor social }\end{array}$ & Poder e influência \\
\hline \multirow{4}{*}{$\begin{array}{l}\text { MOTIVOS DO } \\
\text { COMPORTAMENTO } \\
\text { ORGANIZACIONAL }\end{array}$} & $\begin{array}{l}\text { As organizações } \\
\text { buscam legitimidade }\end{array}$ & Mais valor social & $\begin{array}{l}\text { Mobilização de } \\
\text { recursos }\end{array}$ \\
\hline & & $\begin{array}{l}\text { Conformidade a } \\
\text { critérios externos }\end{array}$ & \begin{tabular}{|l|} 
Controle dos critérios \\
externos
\end{tabular} \\
\hline & $\begin{array}{l}\text { As organizações são } \\
\text { interesses dirigidos }\end{array}$ & $\begin{array}{l}\text { Interesses } \\
\text { institucionalmente } \\
\text { definidos }\end{array}$ & $\begin{array}{l}\text { Interesses políticos e } \\
\text { calculados }\end{array}$ \\
\hline & & Submissão & Não submissão \\
\hline
\end{tabular}

Fonte: Oliver (1988)

Esta comparação serve para dois propósitos. Primeiro, a identificação de aspectos comuns nas suposições destas estruturas demonstra o potencial da perspectiva da dependência de recursos para complementar o conjunto de respostas organizacionais às pressões institucionais. Segundo, a 


\section{FÓRUM - A TEORIA INSTITUCIONAL EM UM CONTEXTO BRASILEIRO: DINÂMICAS DE INOVAÇÃO E IMITAÇÃO - TEORIA INSTITUCIONAL E DEPENDÊNCIA DE RECURSOS NA ADAPTAÇÃO ORGANIZACIONAL: UMA VISÃO COMPLEMENTAR \\ Carlos Ricardo Rossetto - Adriana Marques Rossetto}

divergência no foco entre estas perspectivas salienta que os teóricos institucionais precisam reconhecer as suposições sobre o comportamento organizacional para ratificar a representação passiva e acomodada das organizações. Estas suposições incluem o potencial de variação no grau de escolha, conhecimento, influência e alto interesse que as organizações exibem em resposta a pressões institucionais (OLIVER, 1988; OLIVER, 1991).

De acordo com as duas perspectivas, a escolha organizacional é limitada por uma variedade de pressões externas (MEYER; SCOTT; DEAL, 1983; PFEFFER; SALANCIK, 1978), os ambientes são coletivos e interconectados (DIMAGGIO; POWELL, 1983; PFEFFER; SALANCIK, 1978; POWELL, 1988), e as organizações devem ser sensíveis às demandas e expectativas externas para sobreviverem (MEYER; ROWAN, 1977; PFEFFER; SALANCIK, 1978).

A perspectiva da Dependência de Recursos também enfatiza que cada vez mais as organizações enfrentam numerosas demandas, freqüentemente incompatíveis, de uma variedade de atores externos (PFEFFER, 1982; PFEFFER; SALANCIK, 1978). De acordo com Dimaggio e Powell (1983), Meyer e Rowan (1977), Meyer e Scott (1983) e Zucker (1987), os componentes institucionais que exercem pressões e expectativas incluem não somente o Estado e as profissões, como instituições, mas também grupos de interesses e a opinião pública (SCOTT, 1987a; SCOTT, 1987b).

A perspectiva da Dependência de Recursos tende a enfatizar o ambiente da tarefa, embora esta perspectiva trate também do ambiente social das organizações (PFEFFER; SALANCIK, 1978) e o efeito das pressões do Estado sobre as organizações (PFEFFER, 1972b).

Diferenças de ênfase sobre o ambiente institucional e o da tarefa sugerem diferentes locais de poder externo (aqueles que moldam e reforçam as regras institucionais e crenças versus aqueles que controlam a escassez de recursos) e diferentes processos de ligação entre a organização e o ambiente (trocas e fluxo de recursos versus incorporação e isomorfismo) (SCOTT, 1987b; AHLSTROM et al, 2003).

Estas diferenças conduzem a outras conclusões sobre as respostas apropriadas ao ambiente. Os teóricos institucionais têm enfatizado o valor da conformidade com o ambiente institucional e a exigência de que as organizações devam internalizar regras e normas externas (DIMAGGIO; POWELL, 1983; MEYER; ROWAN, 1977; AHLSTROM et al, 2003; WALKER; CARINI, 2002). Os teóricos da dependência de recursos realçam a necessidade da organização de adaptar-se às incertezas ambientais, lidando com interdependências problemáticas e administrando ativamente o controle do fluxo de recursos (PFEFFER; SALANCIK, 1978).

Estas diferenças no modo apropriado de se relacionar com o ambiente refletem suposições divergentes sobre o grau de escolha, conhecimento e auto-interesse que as organizações possuem para lidar com as restrições externas. A perspectiva da Dependência de recursos enfoca um amplo conjunto de comportamentos de escolha que as organizações podem utilizar para manipular dependências externas. (SCOTT, 1987b; THOMPSON, 1967).

Por comparação, a perspectiva Institucional também é capaz de explicar o comportamento sem escolha no contexto das normas e crenças legitimadas. As organizações conformam-se a crenças ou práticas institucionalizadas quando estas crenças ou práticas são validadas externamente e aceitas pelas organizações (DIMAGGIO, 1988), ou quando sua qualidade de "fato social" torna-se a única maneira 


\section{FÓRUM - A TEORIA INSTITUCIONAL EM UM CONTEXTO BRASILEIRO: DINÂMICAS DE INOVAÇÃO E IMITAÇÃO - TEORIA INSTITUCIONAL E DEPENDÊNCIA DE RECURSOS NA ADAPTAÇÃO ORGANIZACIONAL: UMA VISÃO COMPLEMENTAR \\ Carlos Ricardo Rossetto - Adriana Marques Rossetto \\ concebível, óbvia ou natural de conduzir uma atividade organizacional (BERGER; LUCKMANN, 1967; ZUCKER, 1977, 1987).}

Então, por exemplo, as organizações devem, sem questionar, definir e estruturar suas atividades em torno de funções particulares - vendas, finanças, produção - que refletem classificações institucionalizadas (MEYER; ROWAN, 1977).

Organizações devem agir, não por causa de alguma ligação direta a um resultado organizacional positivo (isto é, maior prestígio ou mais recursos), mas porque isto deveria ser impensável de outro modo. Desta maneira, o comportamento organizacional deve ser dirigido não por processos de mobilização de interesses (DIMAGGIO, 1988), mas por aceitação pré-consciente de valores e práticas institucionalizadas.

Tanto os teóricos da perspectiva Institucional e como os da Dependência de Recursos, por esta razão, assumem que a escolha organizacional é possível dentro do contexto das restrições externas, entretanto, para a perspectiva institucional prepondera a conformidade sobre a resistência, a passividade sobre a atividade e a aceitação pré-consciente sobre a manipulação política em resposta às pressões externas.

Ambas as perspectivas sugerem que as organizações tentam obter estabilidade e legitimidade (DIMAGGIO, 1988; DIMAGGIO; POWELL, 1983; DOWLING; PFEFFER, 1975; MEYER; ROWAN, 1983; PFEFFER; SALANCIK, 1978; ZUCKER, 1986), entretanto, quando considerada a perspectiva institucional, os interesse tendem a ser socialmente e institucionalmente definidos (HININGS; GREENWOOD, 1988; SCOTT, 1987a) e o comportamento auto-interessado tende a ser buscado dentro dos argumentos institucionais, em vez de teorizados explicitamente (DIMAGGIO, 1988).

Os motivos da estabilidade diferem entre as duas perspectivas. A perspectiva Institucional pressupõe a tendência de reprodução ou imitação de estruturas formadas a partir das expectativas das profissões ou de normas coletivas do ambiente institucional (DIMAGGIO; POWELL, 1983; ZUCKER; 1977). Diversos estudos têm demonstrado como fatores institucionais tornam-se transmitidos, sustentados e resistem à mudança através do tempo, como um resultado de conformidade a regras ou expectativas institucionais (TOLBERT, 1985). Em contraposição, os teóricos da dependência de recursos afirmam que a estabilidade é alcançada através do exercício de poder, controle ou negociação de interdependências para alcançar recursos vitais e reduzir a incerteza ambiental.

Os graus e desejos de poder atribuídos à organização, em relação a seu ambiente, ocupam um papel central na explicação das suposições divergentes destas duas perspectivas. A perspectiva da Dependência de Recursos admite alguns graus de controle ou influência sobre os recursos do ambiente ou dos padrões de troca da organização com o propósito de alcançar estabilidade.

Já, as explicações colocadas pela abordagem institucional sobre a reprodução e o isomorfismo enfatizam o papel da conformidade, hábito e convenção, em vez de poder organizacional e controle, em contribuição à estabilidade. O poder tende a ser atribuído ao ambiente institucional e não à organização (isomorfismo coercitivo de DIMAGGIO; POWELL, 1983).

Esta abordagem divergente defendida pelas perspectivas em relação à capacidade das organizações de exercerem poder ou influência sobre as pressões externas é uma importante dimensão para a caracterização das respostas organizacionais ao ambiente. 


\section{FÓRUM - A TEORIA INSTITUCIONAL EM UM CONTEXTO BRASILEIRO: DINÂMICAS DE INOVAÇÃO E IMITAÇÃO - TEORIA INSTITUCIONAL E DEPENDÊNCIA DE RECURSOS NA ADAPTAÇÃO ORGANIZACIONAL: UMA VISÃO COMPLEMENTAR \\ Carlos Ricardo Rossetto- Adriana Marques Rossetto}

Outro ponto convergente entre as perspectivas é a importância da legitimidade com o propósito de demonstrar maior valor social e mobilizar recursos, embora a perspectiva da Dependência de Recursos coloque mais ênfase sobre a instrumentalidade da legitimidade (BENSON, 1975; HINNINGS; GREENWOOD, 1988) e o potencial de controlar ou co-optar, em vez de conformar-se a critérios de comportamento impostos externamente.

De acordo com a teoria institucional, conformidade é útil às organizações a fim de alcançar a probabilidade de sobrevivência. As vantagens de conformidade com as normas e requerimentos institucionais têm sido relatadas na literatura institucional, por exemplo, pelo aumento de prestígio, estabilidade, legitimidade, suporte social, comprometimento interno e externo, acesso a recursos, atração de pessoal, ajuste dentro de categorias administrativas, aceitação em profissões e invulnerabilidade a questionamentos (DIMAGGIO, 1988; DIMAGGIO; POWELL, 1983; MEYER; ROWAN, 1977, 1983; SCOTT; MEYER, 1983; MEYER; SCOTT; DEAL; 1983; SCOTT, 1983; ZUCKER, 1988). A perspectiva da Dependência de recursos, em contraste, elabora a virtude da não conformidade.

Para a perspectiva da Dependência de Recursos, as empresas não respondem meramente a restrições externas e controle através da conformidade. Portanto, uma variedade de estratégias deve ser experimentada para alterar a situação, fazendo com que a organização torne a conformidade menos necessária (PFEFFER, 1982). As vantagens da não conformidade, na perspectiva da Dependência de Recursos, incluem a habilidade de manter discrição ou autonomia sobre as novas contingências e a condição de alterar ou controlar o ambiente em concordância com os objetivos organizacionais.

As duas perspectivas, em virtude de seu foco divergente, têm diferido em suas tipificações sobre a influência organizacional em resposta ao ambiente e na utilização de estratégias de conformidade organizacional com as restrições e demandas externas.

A perspectiva Institucional demonstra como comportamentos de não escolha podem ocorrer e persistir, através do exercício do hábito, convenção, conveniência ou obrigação social, mesmo na ausência da comprovação de que estes comportamentos servem aos interesses próprios da organização ou contribuem para a eficiência ou controle organizacional (TOLBERT, 1985; TOLBERT e ZUCKER, 1983; ZUCKER, 1983).

A perspectiva Institucional também chama atenção para a influência do Estado, da sociedade e das pressões culturais sobre a conformação organizacional não dando ênfase às forças do mercado, à escassez de recursos, ao comportamento organizacional, à história organizacional ou às regras e entendimentos consensuais. Esta perspectiva também explica como a passiva condescendência, em oposição à adaptação estratégica ao ambiente externo, pode contribuir para a sobrevivência de uma organização e, como mitos, significados e valores, em vez de eficiência, autonomia e trocas, devem determinar o comportamento organizacional no contexto das pressões externas.

Apesar destas contribuições substanciais, os teóricos institucionais, em virtude de seu foco, têm limitado sua atenção aos efeitos do ambiente institucional sobre a conformidade e o isomorfismo estrutural e têm desconsiderado o papel da influência e resistência nas relações entre a organização e o ambiente (DIMAGGIO, 1988; LU, 2002; PERROW, 1985; POWELL, 1985). 


\section{FÓRUM - A TEORIA INSTITUCIONAL EM UM CONTEXTO BRASILEIRO: DINÂMICAS DE INOVAÇÃO E IMITAÇÃO - TEORIA INSTITUCIONAL E DEPENDÊNCIA DE RECURSOS NA ADAPTAÇÃO ORGANIZACIONAL: UMA VISÃO COMPLEMENTAR \\ Carlos Ricardo Rossetto - Adriana Marques Rossetto}

Não obstante ao exposto acima, observa-se que a integração das perspectivas institucional e da dependência de recursos é o melhor caminho para a explicação dos processos de adaptação estratégica organizacional.

Ao mesmo tempo em que a perspectiva da Dependência de Recursos oferece convergências e divergências à perspectiva Institucional, ambas operam sob duas diferentes suposições teóricas, mas os teóricos de cada uma parecem estar movendo-se juntos. Por exemplo, Oliver (1991) afirma que os institucionalistas precisam reconhecer o comportamento adaptativo voluntarioso que algumas organizações demostram. Além disso, recentes estudos empíricos empregando a estrutura institucional têm demostrado que as organizações não se adaptam passivamente aos seus ambientes.

Similarmente, os teóricos da dependência de recursos estão reconhecendo os aspectos institucionais do processo de decisão estratégica. Por exemplo, Fredrickson e Laquinto (1989) caracterizam o processo de decisão estratégico como inercial. Também Huff (1980) identificou influências da indústria sobre a reformulação estratégica e sugeriu que o exame dos tomadores de decisão foi demasiadamente limitado. Mais recentemente, Hitt e Tyler (1991) descobriram que as características da indústria influenciam significativamente o processo de decisão estratégica.

Em resumo, estas duas perspectivas parecem oferecer visões comp lementares das decisões estratégicas nos processos de adaptação organizacional. Hrebiniak e Joyce (1985) afirmam que a interação das perspectivas do determinismo e do não determinismo (voluntarismo) devem ser estudadas para o total entendimento do comportamento organizacional.

\section{CONCLUSÕES}

O entendimento do estudo do processo de adaptação estratégica organizacional se consolida cada vez mais a partir das diversas abordagens propostas por teóricos organizacionais. Entretanto, ainda se observa o uso isolado uma determinada teoria para analisar as decisões organizacionais ao longo do tempo da história organizacional não consegue explica-la na sua totalidade.

Este ensaio revisou os pressupostos da Perspectiva Institucional e os da Dependência de Recursos, e identificou um forte potencial de complementaridade entre as abordagens, fato que enriquece em muito o conhecimento já construído sobre os processos de adaptação estratégica organizacional. Apesar das diferenças entre as perspectivas, a ponte de complementaridade identificada, decorre da concepção que elas têm a respeito do meio ambiente, como sendo o fator chave do funcionamento organizacional.

Pode-se observar que, ao buscar visões diferenciadas da influência do ambiente sobre as organizações consegue-se criar um amplo cenário para explicar as distintas estratégias definidas pelos tomadores de decisão, fato que dificilmente ocorreria caso fosse utilizada apenas uma das perspectivas para estudar o processo de adaptação estratégica.

Além disso, as constantes mudanças no ambiente empresarial, fortemente influenciadas pela entrada das grandes corporações nos países em fase de desenvolvimento através da implementação de best 


\section{FÓRUM - A TEORIA INSTITUCIONAL EM UM CONTEXTO BRASILEIRO: DINÂMICAS DE INOVAÇÃO E IMITAÇÃO - TEORIA INSTITUCIONAL E DEPENDÊNCIA DE RECURSOS NA ADAPTAÇÃO ORGANIZACIONAL: UMA VISÃO COMPLEMENTAR \\ Carlos Ricardo Rossetto- Adriana Marques Rossetto}

pratices, têm gerado processos tão complexos e distintos de adaptação das empresas ao ambiente que só podem ser explicadas a partir dos pressupostos das duas perspectivas.

À medida que a investigação organizacional se intensifica no final dos anos 90, tem-se a clareza de que existe a necessidade de utilizar, de maneira combinada, as aplicações das diversas teorias. As duas perspectivas analisadas colocam diferentes racionalidades nas ações organizacionais e oferecem, quando utilizadas em conjunto, um vasto instrumental para análise das organizações. Ambiente externo e interno passam a compor a análise e sua influência pode ser explicada por completo, eliminando espaços de incertezas ou suposições.

Portanto, há que se considerar a visão das duas perspectivas na análise das decisões estratégicas, considerando a extensão em que cada uma delas consegue explicar os diferentes processos de adaptação organizacional.

\section{REFERÊNCIAS}

ABIDIN, Z. Z.; TAYLOR, D. W. Institutional and resources dependency effects on human resouce training and development activity levels of corporation in Malaysia. Journal of American Academy of Business, p. 260-265, set. 2002.

AHLSTROM, D.; YOUNG, M. N.; NAIR, A.; LAW, P. Managing the institutional environment: challenges for foreign firms in post WTO China. Advanced management Journal, v. 68, n. 2, p. 41-49, 2003.

ALDRICH, H. E.; PFEFFER, J. Organizations and envioronments. Ithaca: New Uork State School of Industrial and Labor Relations, Cornell University, 1976.

ALDRICH, H. E. Organizations and environments. Englewood Cliffs, NJ: Prentice-Hall, 1979.

ANDRADE, J. A.; MESQUITA, Z. A certificação de produtos orgânicos e seu processo de institucionalização no brasil. In: ENCONTRO ANUAL DA ASSOCIAÇÃO NACIONAL DE PÓSGRADUAÇÃO EM ADMINISTRAÇÃO, 2003, Atibaia. Anais. Atibaia: ANPAD, 2003. 1 CD-ROM.

ANDREWS, K. R. The concept of corporate strategy. Homewood: Dow Jones-Irwin, 1971.

ASTLEY, W. G.; VAN DE VEN, A. H. Central perspectives and debates in organization theory. Administrative Science Quarterly, v. 28, p. 245-273. 1983.

ALVES, M. A.; KOGA, N. M. Um passo para frente, um passo para trás: a reforma macro legal do terceito setor à luz da teoria institucional. In: ENCONTRO ANUAL DA ASSOCIAÇÃO NACIONAL DE PÓS-GRADUAÇÃO EM ADMINISTRAÇÃO, 2003, Atibaia. Anais. Atibaia: ANPAD, 2003. 1 CD-ROM.

BERGER, P. L.; LUCKMANN, T. The social construction of reality. New York: Doubleday, 1967. 
FÓRUM - A TEORIA INSTITUCIONAL EM UM CONTEXTO BRASILEIRO: DINÂMICAS DE INOVAÇÃO E IMITAÇÃO - TEORIA INSTITUCIONAL E DEPENDÊNCIA DE RECURSOS NA ADAPTAÇÃO ORGANIZACIONAL: UMA VISÃO COMPLEMENTAR

Carlos Ricardo Rossetto - Adriana Marques Rossetto

BENSON, J.K. The interorganizational network as a political economy. Administrative Science Quarterly, v. 20, p. 165-176, 1975.

BOEKER, W. The Permanence of Organizational Strategy. Academy of Management Proceedings, v. 48 , p. 2-6, 1988.

BOEKER, W.; GOODSTEIN, J. Organizational performance and adaptation: effects of environmente and performance on changes in board composition. Academy of Management Journal, v. 34, p. 802826, 1991.

BOURGEOIS, L. J. Strategic mangement and determinism Academy of Management review. v. 9, p. 586-596, 1984.

CARVALHO, C. A. P., VIEIRA, M. M. F.; LOPES, F. D. Perspectiva institucional para análise das organizações. In: ENCONTRO ANUAL DA ASSOCIAÇÃO NACIONAL DE PÓS-GRADUAÇÃO EM ADMINISTRAÇ ÃO, 1999, Foz do Iguaçu. Anais. Foz do Iguaçu: ANPAD, 1999. 1 CD-ROM.

CHAFFEE, E. E. Three Models of Strategy. Academy of Management Review, v.10, p. 89-98, 1985.

CHANDLER, A. D. Strategy and structure: chapters in the history of the american industrial enterprise. Cambridge: Massachusetts Institute of technology Press. 1962.

CHILD, J. Organization structure, environmente, and performance. Sociology. v. 6, p. 12-27, 1972.

COLLINS, R. The credential society. New York: Academic Press, 1979.

CUNHA, C. J. C. A. Adaptação estratégica em ambiente turbulento. 1996. Tese para concurso de professor titular - Programa de Pós-Graduação em Engenharia de Produção - Departamento de Engenharia de Produção e Sistemas, UFSC, Florianópolis, 1996.

DANIELS, K.; JOHNSON, G.; CHERNATONY, L. Task and institutional influences on managers mental models of competition. Organization Studies, v. 23, n. 1, p. 31-62, 2002.

DIMAGGIO, P. J.; POWELL, W. W. The iron cage revisited: institutional isomorphism and collective rationality in organizational fields. American Sociological Review, v. 48, p. 147-160, 1983.

DIMAGGIO, P. J. insterest and agency in institutional theory. In: Lynne G. Zucker (ed.), Institutional patterns and organizations: culture and environments. Cambridge, MA: Ballinger, 1988. p. 3-21.

DOWLING, J. ;PFEFFER, J. Organizational legitimacy. Pacific Sociological Review. v. 18, p. 122$136,1975$.

EISENHARDT, K. M. Agency and institutional theory explanations; the case of retail sale compensation. Academy or Management Journal, v. 31, p. 488-511, 1988.

FENNELL, M. L. The effects of environmental characteristics on the structure of hospital clusters. Administrative Science Quarterly, v. 25, p. 485-510. 1980. 
FÓRUM - A TEORIA INSTITUCIONAL EM UM CONTEXTO BRASILEIRO: DINÂMICAS DE INOVAÇÃO E IMITAÇÃO - TEORIA INSTITUCIONAL E DEPENDÊNCIA DE RECURSOS NA ADAPTAÇÃO ORGANIZACIONAL: UMA VISÃO COMPLEMENTAR

Carlos Ricardo Rossetto - Adriana Marques Rossetto

FREDRICKSON, J. W.; LAQUINTO, A. Inertia and creeping rationality in strategic decision processes. Academy of Management Journal, v. 32, p. 516-542, 1989.

FROOMAN, J. Stakeholder influence strategies. Academy or Management Review, v. 24, n. 2, p. 191$205,1999$.

GINSBERG, A.; BUCHHOLTZ, A. Converting to for-profit status: corporate responsiveness to radical change. Academy of Managemente Jour, v. 33, p. 445-477, 1990.

GREINER, L. E. Evolution and revolution as organizations grow. Harvard BusinessReview, v. 50, p. 37-46, 1972.

HAMBRICK, D. C.; FINKELSTEIN, S. Managerial Discretion: a Bridge Between Polar Views of Organizational Outcomes. In: CUMMINGS, L. L.; STAW, B. M. (Ed.). Research in Organizational Behavior. Greenwich: JAI Press, 1987, p. 369-406.

HANNAN, M. T.; FREEMAN, J. H. The population ecology of organizations. American Journal of Sociology, v. 82, p. 929-964, 1977.

HANNAN, M. T.; FREEMAN, J. H. Structural inertia and organizational change. American Sociological Review, v. 49, p. 149-164, 1984.

HININGS,C. R.; GREENWOOD, R. The dynamics of strategic change. Oxford: Brasil Blackwell, 1988.

HIRSCH, P. M. Processing fads and fashions: an organization set analysis of cultural industry systems. American Journal of Sociology, v. 77, p. 639-659, 1972.

HIRSCH, P. M. Organizational effectiveness and the institucional environment. Administrative Science Quarterly, v. 20, p. 327-344, 1975.

HITT, M. A.; TYLER, B. B. Strategic decision models: integrating different perspectives. Strategic Management Journal, v. 12, p. 327-351, 1991.

HREBINIAK, L. G.; JOYCE, W. F. Organizational adaptation: strategic choice and environmental determinism. Administrative Science Quarterly, v. 30, p. 336-349, 1985.

HUFF, A. S. Organizations as political system: implications for diagnoses, change, and stability. In: CUMMINGS, T. G. Systems theory for organization development. Chichester: John Wiley \& Sons, p. 163-180, 1980.

JENNINGS, D. F.; SEAMAN, S. L. High and low levels of organizational adaptation: an empirical analysis of strategy, structure, and performance. Strategic Management Journal, v. 15, p. 459-475, 1994.

KANTER, R. M. The change masters. New York: Simon and Schuster, 1983.

KELLY, D.; AMBURGEY, T. L. Organizational Inertia and Momentum: A Dinamic Model of Strategic Change. Academy of Management Journal, v. 34, n. 3, p. 591-612, set. 1991. 
FÓRUM - A TEORIA INSTITUCIONAL EM UM CONTEXTO BRASILEIRO: DINÂMICAS DE INOVAÇÃO E IMITAÇÃO - TEORIA INSTITUCIONAL E DEPENDÊNCIA DE RECURSOS NA ADAPTAÇÃO ORGANIZACIONAL: UMA VISÃO COMPLEMENTAR

Carlos Ricardo Rossetto - Adriana Marques Rossetto

LARSON, M. S. The rise of professionalism: a sociological analysis. Berkeley: University of California Press, 1977.

LU, J. W. Intra-and inter-organizational imitative behavior: institutional influences on Japanese firms' entry mode choice. Journal of International Business Studies, v. 33, n. 1, p. 19-37, 2002.

MACHADO-DA-SILVA, C., FONSECA, V. Homogeneização e diversidade organizacional: uma visão integrativa. In: ENCONTRO ANUAL DA ASSOCIAÇÃO NACIONAL DE PÓSGRADUAÇÃO EM ADMINISTRAÇÃO, 1993, Salvador. Anais. Salvador: ANPAD, 1993. 1 CDROM.

MACHADO-DA-SILVA, C., FONSECA, V. S. e FERNANDES, B. H. R. Mudanças e estratégia nas organizações: perspectivas cognitiva e institucional. In: VIEIRA, M. M. F.; OLIVEIRA, L. M. B. (Org.). Administração contemporânea: perspectivas estratégicas. São Paulo: Atlas, 1999.

MACHADO-DA-SILVA, GONÇALVES, S. A. Nota Técnica: A Teoria Institucional. In: MACHADO-DA-SILVA, GONÇALVES, S. A. Handbook de Estudos Organizacionais: Modelos de Análise e Novas Questões em Estudos Organizacionais. São Paulo: Atlas, 1999, cap. 7, p. 220-226.

MCNEIL, K.; MINIHAN, E. Regulation of medical devices and organizational behavior in hospitals. Administrative Science Quarterly, v. 26, p. 475-490, 1977.

MELO, K. V. A.; OLIVEIRA, R. R. Mestrados profissionais: origens, processos de institucionalização e tendências atuais. In: ENCONTRO ANUAL DA ASSOCIAÇÃO NACIONAL DE PÓSGRADUAÇÃO EM ADMINISTRAÇÃO, 2003, Atibaia. Anais. Atibaia: ANPAD, 2003. 1 CD-ROM.

MEYER, J. W. The impact of the centralization of educational funding and control on state and local organizational governance. Stanford, CA: Institute for Research on Educational Finance and Governance, Stanford University, Program Report, 1979.

MEYER, J. W.; ROWAN, B. Institutionalized organizations: formal structure as myth and ceremony. American Journal of Sociology, v. 83, p. 340-363, 1977.

MEYER, J. W.; ROWAN, B. The Structure of Educational Organizations. In: MEYER, J. W.; SCOTT, W. R (Ed.), Organizational environmente: ritual and rationality. Beverly Hills, CA: Sage, 1983. p. 199-215

MEYER, J. W.; SCOTT, W. R. Organizational environmentes: ritual and rationality. Beverly Hills: Sage Publications, 1983.

MEYER, J. W.; SCOTT, W. R.; DEAL, T. E. Institutional and techmical sources of organizational structure of educational organizations. In: MEYER, J. W.; SCOTT, W. R. (Ed.) Organizational environments: ritual and rationality. Beverly Hills, CA: Sage, 1983. p. 45-70

MEYER, J. W. SCOTT, W. R. \& STRANG, D. Centralization, fragmentation and school district complexity. Administrative Science Quaterly, v. 32, p. 186-201, 1987.

MILES, R. H. Macro organizational behavior. Glenview, Illinois: Scot Foresman and Company, 1980. 
FÓRUM - A TEORIA INSTITUCIONAL EM UM CONTEXTO BRASILEIRO: DINÂMICAS DE INOVAÇÃO E IMITAÇÃO - TEORIA INSTITUCIONAL E DEPENDÊNCIA DE RECURSOS NA ADAPTAÇÃO ORGANIZACIONAL: UMA VISÃO COMPLEMENTAR

Carlos Ricardo Rossetto - Adriana Marques Rossetto

MILES, R. H., CAMERON, K. S. Coffin Nails an Corporate Strategies. New Jersey: Prentice Hall inc, Eglewood Cliffs, 1982.

OLIVER, C. The collective strategy framework: an application to competing predictions of isomorphism. Administrative Science Quarterly, v. 33, p. 543-561,1988.

OLIVER, C. Strategic responses to institutional processes. Academy of Management Review, v. 16, p. $145-179,1991$.

PERROW, C. Comment on Langton. Administrative Science Quarterly. v. 30, p. 278-283, 1985.

PFEFFER, J. Size and composition of corporate boards of directors: the organization and its environmente. Administrative Science Quarterly. v. 17, p. 218-228, 1972a.

PFEFFER, J. Merger as a response to organizational interdependence. Administrative Science Quarterly. v. 17, p. 382-394, 1972 b.

PFEFFER, J. Organizations and organization theory. Marshfielld, MA: Pitman, 1982.

PFEFFER, J.; LEBLEBICI, H. The effect of competition on some dimensions of organizational structure. Social Forces. v. 52, p. 268-279, 1973.

PFEFFER, J.; SALANCIK, G. The external control of organizations: a resource dependence perspective. New York: Harper \& Row, 1978.

POWELL, W. W. Getting into print: the decision making process in scholarly publishing. Chicago: University of Chicago Press, 1985.

POWELL, W. W. Institutional effects on organizational structure and performance. In: ZUCKER, L. G. (ed.). Institutional Patterns and Organizations. Cambridge, MA: Ballinger, 1988. p. 115-136.

PRATES, A. A. P. Organização e instituição no velho e novo institucionalismo. In: RODRIGUES, S. B. e CUNHA, M. P. (org.). Novas perspectivas na administração de empresas: uma coletânea lusobrasileira. São Paulo: Iglu, 2000.

REDMOND, W. H. Innovation, diffusion, and institutional change. Journal of Economic Issues, v. 37, n. 3, p. 665-679, 2003.

ROSSETTO, C. R. Adaptação Estratégica Organizacional: Um Estudo Multi-Caso na Indústria da Construção Civil - Setor de Edificações. 1998. 189 f. Tese (Doutorado em Engenharia de Produção) Departamento de Engenharia de Produção e Sistemas, Universidade Federal de Santa Catarina, Florianópolis, 1998.

ROSSETTO, C. R., ROSSETTO, A. M. O Comportamento Estratégico Segundo a Teoria de Miles e Snow: O estudo Multicaso em Três Empresas Familiares na Indústria da Construção Civil - Setor de edificações In: II Encontro de estudos sobre Empreendedorismo e gestão de Pequenas Empresas (EGEPE), 2001, Londrina. Anais. Londrina: EGEPE, 2001, v.1. 
FÓRUM - A TEORIA INSTITUCIONAL EM UM CONTEXTO BRASILEIRO: DINÂMICAS DE INOVAÇÃO E IMITAÇÃO - TEORIA INSTITUCIONAL E DEPENDÊNCIA DE RECURSOS NA ADAPTAÇÃO ORGANIZACIONAL: UMA VISÃO COMPLEMENTAR

Carlos Ricardo Rossetto - Adriana Marques Rossetto

ROSSETTO, C. R., ROSSETTO, A. M. A influência do Isomorfismo como balizador da formulação de estratégias em organizações familiares. In: ENCONTRO ANUAL DA ASSOCIAÇÃO NACIONAL DE PÓS-GRADUAÇÃO EM ADMINISTRAÇ ̃̃O, 2002, Salvador. Anais. Salvador: ANPAD, 2002. 1 CD-ROM.

ROSSETTO, C. R., ROSSETTO, A. M., MELLO, L. F. A influência dos stakeholders no processo de formulação das estratégias organizacionais: O caso da Trevisan Incorporações e Participações Ltda In: CLADEA, 2002, Por to Alegre. Anais. Porto Alegre: CLADEA, 2002, v. 1.

ROSSETTO, C. R., SAlVADOR, E., MELlO, R. B. de. The Strategic Adaptation in Civil Construction Industry: A case Study in Passo Fundo City (RS) In: IGLC-10, 2002, Gramado. Anais. Gramado, IGLC-10, 2002, v.1.

ROSSETTO, C. R., ROSSETTO, A. M. O estudo da formulação estratégica de adaptação organizacional sobre a ótica do modelo de Tushmann e Romanelli In: ENCONTRO ANUAL DA ASSOCIAÇÃO NACIONAL DE PÓS-GRADUAÇÃO EM ADMINISTRAÇÃO, 2003, Atibaia. Anais. Atibaia: ANPAD, 2003, v.1.

ROSSETTO, C. R., CASTRO, J. C. R. Processo de Adaptação estratégica: Um estudo de Caso In: Third International Conference Iberoamerican Academy of Management, 2003, São Paulo. Anais. São Paulo: Third Iberoamenrican, 2003, v.1.

ROWAN, B. Organizational structure and the institucional environmente: the case of public schools. Administrative Science Quarterly. v. 27, p. 259-279, 1982.

SCHENDEL, D.; HOFER, C. Strategic management: a new view of business policy and planning. Boston, MA: Little, Brown, 1979.

SCOTT, W. R. The organization of environmentes: network, cultural and historical elements. In: MEYER, J. W.; SCOTT, W. R. (Ed.). Organizational environments: ritual and rationality. Beverly Hills, CA: Sage, 1983. p. 45-70.

SCOTT, W. R. Organizations: rational, natural, and open systems, 2. ed. Englewood Cliffs, NJ: Prentice Hal, 1987a.

SCOTT, W. R. The adolescence of institutional theory. Administrative Science Quarterly, v. 32, p. 493 $511,1987 \mathrm{~b}$.

SCOTT, W. R.; MEYER, J. The organization of so cietal sectors. In: MEYER, J.; SCOTT, W. R. (eds). Organizational environments. Beverly Hills, CA: Sage, 1983.

SELZNICK, P. TVA and the grass roots. Berkeley: University of california Press. 1949.

SELZNICK, P. Leadership in administration. Evanston: Northwestern University Press, 1957.

SELZNICK, P. Institucionalism "Old" and "New". Administrative Science Quarterly. v. 41, n. 2, p. 270-277, jun. 1996. 
FÓRUM - A TEORIA INSTITUCIONAL EM UM CONTEXTO BRASILEIRO: DINÂMICAS DE INOVAÇÃO E IMITAÇÃO - TEORIA INSTITUCIONAL E DEPENDÊNCIA DE RECURSOS NA ADAPTAÇÃO ORGANIZACIONAL: UMA VISÃO COMPLEMENTAR

Carlos Ricardo Rossetto - Adriana Marques Rossetto

SINGH, J. V.; TUCKER, D. J.; HOUSE, R. J. Organizational change and organizational mortality. Administrative Science Quarterly, v. 31, p. 587-611, 1986.

STARBUCK, W. H. Organizational metamorphosis. In: STARBUCK W. H. (Ed.). Organizational growth and development. Baltimore: Penguin Books, 1971, p. 275-298.

STATA, N. M.; PHAN, D. D. E-business success at intel: am organization ecology and resource dependence perspective. Industrial Management Data Systems, v. 102, n.3/4, p. 211-217, 2002.

THOMPSON, J. Organizations in action. New York: Jonh Wiley, 1967.

TOLBERT, P. S.; ZUCKER, L. G. Institutional sources of change in the formal structure of organizations: the diffusion of civil sevice reforms, 1880-1935. Administrative Science Quarterly, v. 28, p. 22-39, 1983.

TOLBERT, P. S. Institutional environments and resource dependence: sources of administrative structure in institutions of higher learning. Administrative Science Quarterly, v. 30, p. 1-13, 1985.

WALKER, G. M. L. T.; CARINI, G. How does institutional change affect heterogeneity among firms? Strategic Managemente Journal. v. 23, p. 89-104, 2002.

ZAJAC, E.; SHORTELL, S. M. Changing generic strategies: likelihood, direction, and performance implications. Strategic Managemente Journal. v. 10, p. 413-430, 1989.

ZALD, M. The social control of industries. Social Forces, v. 57, p. 79-102, 1978.

ZUCKER, L. G. The role of institutionalization in cultural persistence. American Sociological Review . v. 42, p. 726-743, 1977.

ZUCKER, L. G. Organizations as institutions. In: BACHARACH, S. (ed.). Research in the Sociology of Organizations. Greenwich, Conn.: JAI Press, 1983. p. 1-47.

ZUCKER, L. G. The production of trust: institutional sources of economic structure, 1840-1920. In: STAW, B.; CUMMINGS, L. L. (Ed.). Research in organizational behavior. Greenwich, CT: JAI, 1986. p. 53-112.

ZUCKER, L. G. Institutional theories of organization. Annual Rewiew of Sociolog, v. 13, p. 443-464, 1987.

ZUCKER, L. G. Institutional patterns and organizations. Cambridge, MA: Ballinger, 1988.

ZAJAC, E.; SHORTELL, S. M. Changing generic strategies: likelihood, direction, and performance implications. Strategic Managemente Journal, v. 10, p. 413-430, 1989. 
FÓRUM - A TEORIA INSTITUCIONAL EM UM CONTEXTO BRASILEIRO: DINÂMICAS DE INOVAÇÃO E IMITAÇÃO - TEORIA INSTITUCIONAL E DEPENDÊNCIA DE RECURSOS NA ADAPTAÇÃO ORGANIZACIONAL: UMA VISÃO COMPLEMENTAR

Carlos Ricardo Rossetto - Adriana Marques Rossetto

Artigo recebido em 01.03.2003. Aprovado em 16.06.2004.

\section{Carlos Ricardo Rossetto}

Coordenador e Professor do Programa de Mestrado Acadêmico em Administração da UNIVALI. Doutor em Engenharia de produção.

Interesses de pesquisa nas áreas de estratégia empresarial, mudança organizacional e teoria institucional

E-mail: rossetto@big.univali.br

Endereço: Rua das Acácias, 121, bloco B 3, apto 402, Carvoeira, Florianópolis - SC, 88040-560.

\section{Adriana Marques Rossetto}

Professora do Mestrado em Gestão de Políticas Públicas da UNIVALI. Doutora em Engenharia de Produção.

Interesses de pesquisa nas áreas de planejamento estratégico, teoria institucionale gestão pública. E-mail: arossetto@univali.br

Endereço: Rua das Acácias, 121, bloco B 3, apto 402, Carvoeira, Florianópolis - SC, 88040-560. 